\title{
Digital Strategies to a Local Cultural Tourism Development: Project e- Carnide
}

Tourism, Culture and Heritage in a Smart Economy pp 365-383

- Authors

- Authors and affiliations

- Maria Isabel Roque

- Maria João Forte

- Maria Isabel Roque

○ 1

○ 2

- Maria João Forte

○ 1

1. 1.School of Tourism, Sport and HospitalityEuropeia

UniversityLisbonPortugal

2. 2.CIDEHUS, Évora UniversityÉvoraPortugal

Conference paper

First Online:

22 February 2017

DOI (Digital Object Identifier): 10.1007/978-3-319-47732-9_24

- 32Downloads

Part of the book series Springer Proceedings in Business and Economics (SPBE)

Cite this paper as:

Roque M.I., Forte M.J. (2017) Digital Strategies to a Local Cultural

Tourism Development: Project e-Carnide. In: Katsoni V., Upadhya A.,

Stratigea A. (eds) Tourism, Culture and Heritage in a Smart Economy.

Springer Proceedings in Business and Economics. Springer, Cham

\begin{abstract}
Digital humanities and smart economy strategies are being seen as an important link between tourism and cultural heritage, as they may contribute to differentiate the audiences and to provide different approaches. Carnide is a peripheral neighbourhood of Lisbon with an elderly population, visible traces of rurality, and strong cultural and religious traditions. The academic project e-Carnide concerns its tangible and intangible cultural heritage and the data dissemination through a website and a mobile app, with textual and visual information. The project aims to analyse the impact of technological solutions on cultural tourism development in a sub-region, involving interdisciplinary research in heritage, history of art, ethnography, design communication and software engineering and the collaboration between the university and local residents in a dynamic and innovative way. Framed by a theoretical approach about the role of smart economy for the cultural tourism development in peripheral areas, this paper focuses on a case study, dealing with documents, interviews and observations, in order to understand how the e-Carnide project evolves. The study comprises an analysis about the strengths, weaknesses, opportunities and threats (SWOT analysis) of the project in view to realize its social and cultural implications and to appreciate how it can be applied in other similar and enlarged projects. Results of
\end{abstract}


the research indicates that the new technological strategies can promote the involvement of the population in the knowledge of its own heritage as a factor of cultural and creative tourism development centred on an authentic and immersive experience of the places.

\section{Keywords}

Cultural tourism Heritage Peripheral area Smart tourism Digital humanities JEL Classification

Z320 tourism and development

\section{References}

1. Arp, J. (1990). Residents' perceptions research on the social impacts of tourism.

Annals of Tourism Research, 17(4), 610-616. doi:10.1016/0160-7383(90)90032-M

(http://dx.doi.org/10.1016/0160-7383(90)90032-M).CrossRef

(http://dx.doi.org/10.1016/0160-7383(90)90032-M)Google Scholar

(http://scholar.google.com/scholar_lookup?

title=Residents\%E2\%80\%99\%20perceptions\%2oresearch\%20on\%20the\%20social \%20impacts\%20of\%2otourism\&author=J.\%20Arp\&journal=Annals\%20of\%20Tou rism\%20Research\&volume $=17$ \&issue $=4 \&$ pages $=610-$

616\&publication_year $=1990 \& d o i=10.1016 \% 2 F 0160-7383 \% 2890 \% 2990032-\mathrm{M}$ )

2. Ashworth, G. J., Graham, B. J., \& Tunbridge, J. E. (2007). Pluralising pasts:

Heritage, identity and place in multicultural societies. London, UK: Pluto

Press.Google Scholar (http://scholar.google.com/scholar_lookup?

title=Pluralising\%20pasts\%3A\%20Heritage\%2C\%2oidentity\%20and\%2oplace\%20i n\%20multicultural\%20societies\&author=GJ.\%20Ashworth\&author=BJ.\%20Graha m\&author=JE.\%20Tunbridge\&publication_year=2007)

3. Barbini, F. M., \& Presutti, M. (2014). Transforming a peripheral area in an emerging tourism destination. Tourism Geographies, 16(2), 190-206. doi:10.1080/14616688.2014.888589

(http://dx.doi.org/10.1080/14616688.2014.888589).CrossRef

(http://dx.doi.org/10.1080/14616688.2014.888589)Google Scholar

(http://scholar.google.com/scholar_lookup?

title=Transforming $\% 20 a \%$ 2operipheral\%20area $\% 20$ in $\% 20 a n \% 20$ emerging $\%$ 20to urism\%2odestination\&author=FM.\%20Barbini\&author=M.\%20Presutti\&journal=

Tourism\%20Geographies\&volume $=16 \&$ issue $=2 \&$ pages $=190-$

206\&publication year $=2014 \&$ doi $=10.1080 \% 2 \mathrm{~F} 14616688.2014 .888589)$

4. Benton, T. (2010). Understanding heritage and memory. Manchester, UK:

Manchester University Press, Open University.Google Scholar

(http://scholar.google.com/scholar_lookup?

title=Understanding\%2oheritage\%20and\%20memory\&author=T.\%2oBenton\&pub lication_year=2010)

5. Berg, B. L. (2001). Qualitative research methods for the social sciences (8th ed.).

Boston, MA: Allyn and Bacon.Google Scholar

(http://scholar.google.com/scholar lookup?

title=Qualitative\%20research\%20methods\%2ofor\%20the\%20social\%20sciences\&a uthor=BL.\%2oBerg\&publication_year $=2001)$

6. Besculides, A., Lee, M. E., \& McCormick, P. J. (2002). Residents' perceptions of the cultural benefits of tourism. Annals of Tourism Research, 29(2), 303-319. doi:10.1016/So16o-7383(01)00066-4 (http://dx.doi.org/10.1016/So1607383(01)ooo66-4).CrossRef (http://dx.doi.org/10.1016/So160-7383(01)ooo664)Google Scholar (http://scholar.google.com/scholar_lookup? title=Residents\%E2\%80\%99\%20perceptions\%200f\%20the\%20cultural\%2obenefit s\%20of\%2otourism\&author=A.\%20Besculides\&author=ME.\%2oLee\&author=PJ.\% 20McCormick\&journal=Annals\%20of\%20Tourism\%20Research\&volume=29\&issu $\mathrm{e}=2$ \&pages $=303-319 \&$ publication_year $=2002 \& d o i=10.1016 \% 2 F S 0160-$ 7383\%2801\%2900066-4)

7. Blomgren, K. B., \& Sørensen, A. (1998). Peripherality: Factor or feature? reflections on peripherality in tourism research. Progress in Tourism and Hospitality Research, 4(4), 319-336. doi:10.1002/(SICI)1099-1603(199812)4:4<319:AIDPTH134>3.0.CO;2-V (http://dx.doi.org/10.1002/(SICI)10991603(199812)4\%3A4\%3c319\%3AAID-PTH134\%3e3.0.CO\%3B2-V).CrossRef (http://dx.doi.org/10.1002/(SICI)1099-1603(199812)4\%3A4<319\%3A\%3AAIDPTH134>3.0.CO\%3B2-V)Google Scholar (http://scholar.google.com/scholar_lookup? title=Peripherality\%3A\%20Factor\%20or\%2ofeature\%3F\%2oreflections $\% 200$ \%20 peripherality\%2oin\%20tourism\%20research\&author=KB.\%20Blomgren\&author= A.\%20S\%C3\%B8rensen\&journal=Progress\%20in\%20Tourism\%2Oand\%20Hospital ity $\% 20$ Research $\&$ volume $=4 \&$ issue $=4 \&$ pages $=319-$

$336 \&$ publication year $=1998 \&$ doi $=10.1002 \% 2$ F\%28SICI\%291099-

1603\%28199812\%294\%3A4\%3C319\%3A\%3AAID-PTH134\%3E3.0.CO\%3B2-V)

8. Boniface, P., \& Fowler, P. J. (1993). Heritage and tourism in "the global village". London, UK: Routledge.Google Scholar

(http://scholar.google.com/scholar_lookup?

title=Heritage\%20and\%20tourism\%20in\%20\%E2\%80\%9Cthe\%2oglobal\%2ovillag 
e\%E2\%80\%9D\&author=P.\%20Boniface\&author=PJ.\%2OFowler\&publication_year =1993)

9. Brown, F., \& Hall, D. R. (2000). Tourism in peripheral areas: Case studies.

Buffalo, NY: Channel View Publications.Google Scholar

(http://scholar.google.com/scholar_lookup?

title=Tourism\%20in\%2operipheral\%20areas\%3A\%20Case\%20studies\&author=F .

$\%$ 20Brown\&author=DR.\%20Hall\&publication_year=2000)

10. Buhalis, D., \& Amaranggana, A. (2014). Smart tourism destinations. In I.

Tussyadiah \& A. Inversini (Eds.), Information and communication technologies in tourism 2014: Proceedings of the International Conference in Dublin, Ireland, January 21-24, 2014 (pp. 553-564). Cham, DE: Springer.Google Scholar (http://scholar.google.com/scholar lookup? title=Smart\%20tourism\%20destinations\&author=D.\%20Buhalis\&author=A.\%20A maranggana\&pages $=553-564$ \&publication year $=2014$ )

11. Burnett, K. A. (2001). Heritage, authenticity and history. In S. Drummond \& I. Yeoman (Eds.), Quality issues in heritage visitor attractions (pp. 39-53). Oxford, UK: Butterworth Heinemann.CrossRef (http://dx.doi.org/10.1016/B978-0-75064675-8.50010-1)Google Scholar (http://scholar.google.com/scholar_lookup? title=Heritage $\% 2 \mathrm{C} \% 20$ authenticity\%20and\%2ohistory\&author=KA.\%2oBurnett\&p ages $=39-53 \&$ publication_year $=2001$ )

12. Cameron, F., \& Kenderdine, S. (2007). Theorizing digital cultural heritage: $A$ critical discourse. Cambridge, MA: MIT Press.CrossRef

(http://dx.doi.org/10.7551/mitpress/9780262033534.001.0001)Google Scholar (http://scholar.google.com/scholar_lookup?

title=Theorizing\%20digital\%20cultural\%2oheritage $\% 3$ A\%20A\%20critical\%20disco urse\&author=F.\%20Cameron\&author $=$ S.\%20Kenderdine\&publication_year $=2007$ )

13. Christaller, W., \& Baskin, C. W. (1966). Central places in southern Germany.

Englewood Cliffs, NJ: Prentice-Hall.Google Scholar

(http://scholar.google.com/scholar_lookup?

title=Central\%20places\%20in\%20southern\%20Germany\&author=W.\%20Christall er\&author $=$ CW.\%2oBaskin\&publication_year $=1966$ )

14. Cipolla, F. F. V., Castro, L. C., Nicol, E., Kratky, A., \& Cipolla-Ficarra, M. (2011). Human-Computer Interaction, Tourism and Cultural Heritage: First International Workshop, HCITOCH 2010, Brescello, Italy, September 7-8, 2010 : Revised Selected Papers. Berlin, Heidelberg, DE: Springer, Berlin Heidelberg.Google Scholar (http://scholar.google.com/scholar_lookup? title=Human-

Computer\%2OInteraction\%2C\%20Tourism\%20and\%2oCultural\%2oHeritage\%3A \%20First\%2OInternational\%2oWorkshop\%2C\%2OHCITOCH\%2O2O10\%2C\%2OBre scello\%2C\%2oItaly\%2C\%20September\%207\%E2\%80\%938\%2C\%202010\%3A\%20 Revised\%20Selected\%20Papers\&author=FFV.\%20Cipolla\&author=LC.\%20Castro \&author=E.\%20Nicol\&author=A.\%20Kratky\&author=M.\%20CipollaFicarra\&publication year $=2011)$

15. Cohen D., \& Crabtree, B. (2006). Qualitative research guidelines project: Informal interviewing. Robert Wood Johnson Foundation. Retrieved from http://www.qualres.org/HomeInfo-3631.html (http://www.qualres.org/HomeInfo-3631.html).

16. Council of Europe, (2012). Guidelines on cultural heritage technical tools for heritage conservation and management. Retrieved from https://www.coe.int/t/dg4/cultureheritage/cooperation/Kosovo/Publications/GuidelinesENG.pdf

(https://www.coe.int/t/dg4/cultureheritage/cooperation/Kosovo/Publications/Gu idelines-ENG.pdf).

17. Dear, M., \& Flusty, S. (1999). Engaging postmodern urbanism. Urban Geography, 2O(5), 412-416. doi:10.2747/0272-3638.20.5.412

(http://dx.doi.org/10.2747/0272-3638.20.5.412).CrossRef

(http://dx.doi.org/10.2747/0272-3638.20.5.412)Google Scholar

(http://scholar.google.com/scholar_lookup?

title=Engaging\%2opostmodern\%20urbanism\&author=M.\%2oDear\&author=S.\%20 Flusty\&journal $=$ Urban\%20Geography\&volume $=20 \&$ issue $=5 \&$ pages $=412-$ 416\&publication_year $=1999 \& d o i=10.2747 \% 2$ Fo272-3638.20.5.412)

18. Dickinson, J., Ghali, K., Cherrett, T., Speed, C., Davies, N., \& Norgate, S. H. (2014). Tourism and the smartphone app: Capabilities, emerging practice, and scope in the travel domain. Current Issues in Tourism, 17(1), 84-101. doi:10.1080/13683500.2012.718323

(http://dx.doi.org/10.1080/13683500.2012.718323).CrossRef

(http://dx.doi.org/10.1080/13683500.2012.718323)Google Scholar

(http://scholar.google.com/scholar lookup?

title=Tourism\%20and\%20the\%20smartphone\%20app\%3A\%20Capabilities\%2C\%2 oemerging\%2opractice\%2C\%20and\%20scope\%20in\%2othe\%2otravel\%2odomain \&author=J.\%20Dickinson\&author=K.\%20Ghali\&author=T.\%20Cherrett\&author= C.\%20Speed\&author=N.\%20Davies\&author $=$ SH.\%2ONorgate\&journal=Current\%2 oIssues \%20in $\% 20$ Tourism\&volume $=17 \&$ issue $=1 \&$ pages $=84^{-}$ $101 \&$ publication_year $=2014 \&$ doi $=10.1080 \% 2 \mathrm{~F} 13683500.2012 .718323$ )

19. DigiCULT, (2003) Europe: Creating cooperation for digitization. Retrieved from www.cordis.lu/ist/ka3/digicult/eeurope-overview.htm

(http://www.cordis.lu/ist/ka3/digicult/eeurope-overview.htm).

20. Economou, M. (2015). Heritage in the digital age. In W. Logan, M. N. Craith \& U. Kockel (Eds.), A companion to heritage studies (pp. 215-228). Chichester, West Sussex, UK, Malden, MA: Wiley.CrossRef 
(http://dx.doi.org/10.1002/9781118486634.ch15)Google Scholar

(http://scholar.google.com/scholar_lookup?

title=Heritage\%20in\%20the\%20digital\%20age\&author=M.\%2oEconomou\&pages= 215-228\&publication_year=2015)

21. European Commission, Directorate-General Information Society. (2002). The

DigiCULT Report: Technological landscapes for tomorrow's cultural economy:

Unlocking the value of cultural heritage. Luxembourg, LU: Office for Official

Publications of the European Communities.Google Scholar

(http://scholar.google.com/scholar_lookup?

title=The\%2oDigiCULT\%20Report\%3A\%20Technological\%2olandscapes\%2ofor\% 20tomorrow\%E2\%80\%99s\%20cultural\%20economy\%3A\%20Unlocking\%20the\%2 ovalue\%20of\%20cultural\%20heritage\&publication year=2002)

22. Evans, G. (1998). Urban leisure: Edge city and the new leisure periphery. In M. F.

Collins \& I. S. Cooper, Leisure management: Issues and applications (pp. 113-137). Wallingford, UK, New York, NY: CAB International.

23. Gorman, G. E., \& Clayton, P. (2005). Qualitative research for the information professional: A practical handbook (2nd ed.). London, UK: Facet.Google Scholar (http://scholar.google.com/scholar_lookup?

title=Qualitative\%20research\%2ofor\%20the\%2oinformation\%2oprofessional\%3A \%20A\%20practical\%20handbook\&author=GE.\%20Gorman\&author=P.\%20Clayto n\&publication_year=2005)

24. Gretzel, U., Reino, S., Kopera, S., \& Koo, C. (2015). Smart tourism challenges. Journal of Tourism, 16(1), 41-47.

25. Hall, C. M. (2007). North-South perspectives on tourism, regional development and peripheral areas. In D. Müller \& B. Jansson (Eds.), Tourism in peripheries: Perspectives from the far North and South (pp. 19-27). Wallingford, UK:

Cabi.CrossRef (http://dx.doi.org/10.1079/9781845931773.0019)Google Scholar (http://scholar.google.com/scholar_lookup?title=North-

South\%20perspectives\%20on\%20tourism\%2C\%2oregional\%2odevelopment\%20a nd\%2operipheral\%20areas\&author=CM.\%20Hall\&pages $=19-$ 27\&publication year $=2007$ )

26. Hall, C. M., \& Page, S. (2002). The geography of tourism and recreation: Environment, place and space (2nd ed.). London, UK: Routledge.Google Scholar (http://scholar.google.com/scholar_lookup? title=The\%20geography\%20of\%2otourism\%20and\%2orecreation\%3A\%2oEnviron ment\%2C\%20place\%20and\%20space\&author=CM.\%20Hall\&author=S.\%2OPage\& publication year=2002)

27. Hall, C. M., Harrison, D., Weaver, D., \& Wall, G. (2013). Vanishing peripheries: Does tourism consume places? Tourism Recreation Research, 38(1), 71-92. doi:10.1080/02508281.2013.11081730 (http://dx.doi.org/10.1080/02508281.2013.11081730).CrossRef (http://dx.doi.org/10.1080/02508281.2013.11081730)Google Scholar (http://scholar.google.com/scholar lookup?

title=Vanishing\%20peripheries\%3A\%20Does\%2otourism\%20consume\%2oplaces \%3F\&author=CM.\%20Hall\&author=D.\%20Harrison\&author=D.\%20Weaver\&auth or $=$ G. $\% 20$ Wall\&journal $=$ Tourism\%20Recreation\%20Research\&volume $=38 \&$ issue $=1 \&$ pages $=71-$

92\&publication_year $=2013 \&$ doi $=10.1080 \% 2 \mathrm{Fo} 2508281.2013 .11081730)$

28. Hall, C. M., \& Boyd, S. (Eds.). (2005). Tourism and nature-based tourism in peripheral areas: Development or disaster. Clevedon, UK: Channel View.Google Scholar (http://scholar.google.com/scholar_lookup?

title=Tourism\%20and\%20nature-

based\%20tourism\%20in\%20peripheral\%20areas\%3A\%2oDevelopment\%20or\%20 disaster\&publication_year=2005)

29. Harrison, R. (2010). The politics of heritage. In R. Harrison (Ed.), Understanding the politics of heritage (pp. 154-196). Manchester, UK: Manchester University Press.Google Scholar (http://scholar.google.com/scholar_lookup? title=The\%20politics\%20of\%2oheritage\&author $=$ R.\%20Harrison\&pages $=154-$ 196\&publication_year $=2010)$

30. Hede, A., \& Thyne, M. (2010). A journey to the authentic: Museum visitors and their negotiation of the inauthentic. Journal of Marketing Management, 26(7/8), 686-705. doi:10.1080/02672571003780106

(http://dx.doi.org/10.1080/02672571003780106).CrossRef

(http://dx.doi.org/10.1080/02672571003780106)Google Scholar

(http://scholar.google.com/scholar_lookup?

title=A\%20journey\%20to\%20the\%20authentic\%3A\%2oMuseum\%20visitors\%20a nd\%20their\%2onegotiation\%200\%\%20the\%2oinauthentic\&author=A.\%20Hede\&au thor=M.\%20Thyne\&journal=Journal\%20of\%20Marketing\%2oManagement\&volu $\mathrm{me}=26 \&$ issue $=7 \% 2 \mathrm{~F} 8 \&$ pages $=686-$

705\&publication year $=2010 \&$ doi $=10.1080 \% 2 F 02672571003780106)$

31. Hohl, A., \& Tisdell, C. (1995). Peripheral tourism: Development and management. Annals of Tourism Research, 22(3), 517-534. doi:10.1016/0160-7383(95)00005-Q (http://dx.doi.org/10.1016/0160-7383(95)ooo05-Q).CrossRef (http://dx.doi.org/10.1016/0160-7383(95)00005-Q)Google Scholar (http://scholar.google.com/scholar_lookup? title=Peripheral\%20tourism\%3A\%20Development\%20and\%2omanagement\&auth or=A.\%20Hohl\&author=C.\%20Tisdell\&journal=Annals\%20of\%20Tourism\%20Res earch \&volume $=22 \&$ issue $=3 \&$ pages $=517-$

534\&publication_year=1995\&doi=10.1016\%2F0160-7383\%2895\%2900005-Q) 
32. Jenkins, C. L. (2015). Tourism policy and planning for developing countries: some critical issues. Tourism Recreation Research, 4O(2), 144-156.

doi:10.1080/02508281.2015.1045363

(http://dx.doi.org/10.1080/02508281.2015.1045363).CrossRef

(http://dx.doi.org/10.1080/02508281.2015.1045363)Google Scholar

(http://scholar.google.com/scholar_lookup?

title=Tourism\%20policy\%20and\%2oplanning\%2ofor\%2odeveloping\%2ocountries \%3A\%20some\%20critical\%2oissues\&author=CL.\%20Jenkins\&journal=Tourism\%2 oRecreation $\% 20$ Research\&volume $=40 \&$ issue $=2$ \&pages $=144^{-}$

$156 \&$ publication_year $=2015 \& d$ doi $=10.1080 \% 2 F 02508281.2015 .1045363$ )

33. Jorgensen, D. L. (1989). Participant observation: A methodology for human studies (1st ed.). Newbury Park, CA: Sage Publications.CrossRef

(http://dx.doi.org/10.4135/9781412985376)Google Scholar

(http://scholar.google.com/scholar lookup?

title=Participant\%20observation\%3Ā \%20A\%2omethodology\%2ofor\%2ohuman\%2 ostudies\&author=DL.\%20Jorgensen\&publication_year=1989)

34. Kalay, Y. E., Kvan, T., \& Affleck, J. (2008). New heritage: New media and cultural heritage. London: Routledge.Google Scholar

(http://scholar.google.com/scholar_lookup?

title=New\%2oheritage\%3A\%20New\%20media\%20and\%20cultural\%2oheritage\&a uthor=YE.\%20Kalay\&author=T.\%20Kvan\&author=J.\%20Affleck\&publication_year $=2008$ )

35. Karp, I. \& Lavine, S. (Eds.). (1991). Exhibiting cultures: The poetics and politics of museum display (1st ed.). Washington, WA: Smithsonian Institution Press.Google Scholar (http://scholar.google.com/scholar_lookup?

title=Exhibiting\%20cultures\%3A\%20The\%2opoetics\%20and\%2opolitics\%20of\%2 omuseum\%20display\&publication_year=1991)

36. King, B., Pizam, A., \& Milman, A. (1993). Social impacts of tourism: Host perceptions. Annals of Tourism Research, 2O(4), 650-665. doi:10.1016/01607383(93)90089-L (http://dx.doi.org/10.1016/0160-7383(93)90089-L).CrossRef (http://dx.doi.org/10.1016/0160-7383(93)90089-L) Google Scholar (http://scholar.google.com/scholar_lookup? title=Social\%2oimpacts\%20of\%20tourism\%3A\%20Host\%2operceptions\&author= B.\%20King\&author=A.\%20Pizam\&author=A.\%20Milman\&journal=Annals\%20of\% 20Tourism $\% 20$ Research \&volume $=20 \&$ issue $=4 \&$ pages $=650-$ 665\&publication_year=1993\&doi=10.1016\%2Fo160-7383\%2893\%2990089-L)

37. Kirshenblatt-Gimblett, B. (1998). Destination culture: Tourism, museums, and heritage (1st ed.). Berkeley, CA: University of California Press.Google Scholar (http://scholar.google.com/scholar_lookup?

title=Destination\%20culture\%3A\%20Tourism\%2C\%2omuseums\%2C\%2oand\%2oh eritage\&author=B.\%20Kirshenblatt-Gimblett\&publication_year=1998)

38. Labadi, S., \& Long, C. (Eds.). (2010). Heritage and globalisation. Milton Park, UK, New York, NY: Routledge.Google Scholar

(http://scholar.google.com/scholar_lookup? title=Heritage $\% 20$ and $\% 20$ globalisation\&publication year $=2010$ )

39. Lazzarotti, O. (2003). Tourisme et patrimoine: ad augusta per angustia. Annales de Géographie, 112(629), 91-110. doi:10.3406/geo.2003.893

(http://dx.doi.org/10.3406/geo.2003.893).CrossRef

(http://dx.doi.org/10.3406/geo.2003.893)Google Scholar

(http://scholar.google.com/scholar_lookup?

title=Tourisme\%20et\%20patrimoine\%3A\%20ad\%20augusta\%2oper\%2oangustia\& author=O.\%2oLazzarotti\&journal=Annales\%20de\%20G\%C3\%A9ographie\&volume $=112 \&$ issue $=629 \&$ pages $=91$ -

110\&publication_year $=2003 \&$ doi $=10.3406 \% 2 F g e 0.2003 .893)$

40. Lazzarotti, O. (2011). Patrimoine et tourisme: Histoire, lieux, acteurs, enjeux. Paris, FR: Belin.Google Scholar (http://scholar.google.com/scholar_lookup? title=Patrimoine\%20et\%20tourisme\%3A\%20Histoire\%2C\%2olieux\%2C\%2Oacteur $\mathrm{s} \% 2 \mathrm{C} \%$ 20enjeux\&author $=0 . \% 20$ Lazzarotti\&publication_year $=2011$ )

41. Lengkeek, J. (2008). The authenticity discourse of heritage. In D. J. Breeze \& S. Jilek (Eds.), Frontiers of the Roman empire: The European dimension of a world heritage site (pp. 37-53). Edinburgh, UK: Historic Scotland.Google Scholar (http://scholar.google.com/scholar_lookup?

title=The\%20authenticity\%20discourse\%200f\%2oheritage\&author=J.\%2oLengkee k\&pages $=37-53 \&$ publication_year $=2008$ )

42. Logan, W., Craith, M. N., \& Kockel, U. (Eds.). (2015). A companion to heritage studies. Chichester, West Sussex, UK, Malden, MA: Wiley-Blackwell.Google Scholar (http://scholar.google.com/scholar_lookup?

title=A\%20companion\%20to\%2oheritage\%20studies\&publication year=2015)

43. Lombardo, V., \& Damiano, R. (2012). Storytelling on mobile devices for cultural heritage. New Reviwe of Hipermedia and Multimedia, 18(1-2), 11-15. doi:10.1080/13614568.2012.617846 (http://dx.doi.org/10.1080/13614568.2012.617846).CrossRef (http://dx.doi.org/10.1080/13614568.2012.617846)Google Scholar (http://scholar.google.com/scholar_lookup?

title=Storytelling\%20on\%20mobile\%2odevices\%2ofor\%2ocultural\%2oheritage\&au thor=V.\%20Lombardo\&author=R.\%20Damiano\&journal=New\%20Reviwe\%20of\% 20Hipermedia\%20and\%20Multimedia\&volume=18\&issue=1\%E2\%80\%932\&pages $=11-15 \&$ publication_year $=2012 \& d o i=10.1080 \% 2 \mathrm{~F} 13614568.2012 .617846$ )

44. Lundgren, J. (1982). The tourist frontier of Nouveau Quebec: Functions and regional linkages. Tourist Review, 37(2), 10-16. doi:10.1108/ebo57856 
(http://dx.doi.org/10.1108/eb057856).CrossRef

(http://dx.doi.org/10.1108/eb057856)Google Scholar

(http://scholar.google.com/scholar_lookup?

title=The\%20tourist\%2ofrontier\%200f\%20Nouveau\%20Quebec\%3A\%20Functions

\%20and\%2oregional\%2olinkages\&author=J.\%2oLundgren\&journal=Tourist\%2OR eview\&volume $=37 \&$ \&issue $=2 \&$ pages $=10-$

16\&publication_year $=1982 \&$ doi $=10.1108 \% 2$ Feb057856)

45. MacCannell, D. (1999). The tourist: A new theory of the leisure class (1st ed.). Berkeley, CA. [etc.]: University of California Press.

46. Mazón, T., Huete, R., \& Mantecón, A. (2009). Tourism dependence and host community perceptions: Notes on the social exchange theory. Revista Encontros Científicos: Tourism \& Management Studies, 5, 25-36.Google Scholar

(http://scholar.google.com/scholar_lookup?

title=Tourism\%20dependence\%20and\%2ohost\%20community\%2operceptions\%3 A\%20Notes\%200n\%20the\%20social\%20exchange\%2otheory\&author=T.\%20Maz \%C3\%B3n\&author=R.\%2oHuete\&author=A.\%20Mantec\%C3\%B3n\&journal=Revis ta\%20Encontros\%20Cient\%C3\%ADficos\%3A\%20Tourism\%20\%26\%20Manageme nt\%20Studies\&volume $=5$ \&pages $=25-36 \&$ publication_year $=2009$ )

47. McIntosh, A. J., \& Prentice, R. (1999). Affirming authenticity: Consuming cultural heritage. Annals of Tourism Research, 26(3), 589-612. doi:10.1016/So1607383(99)ooo10-9 (http://dx.doi.org/10.1016/So160-7383(99)ooo10-9).CrossRef (http://dx.doi.org/10.1016/So160-7383(99)oo010-9)Google Scholar

(http://scholar.google.com/scholar_lookup? title=Affirming\%20authenticity\%3A\%20Consuming\%20cultural\%2oheritage\&auth or=AJ.\%20McIntosh\&author=R.\%20Prentice\&journal=Annals\%20of\%20Tourism $\%$ 20Research\&volume $=26 \&$ issue $=3 \&$ pages $=589$ -

612\&publication_year=1999\&doi=10.1016\%2FSo160-7383\%2899\%2900010-9)

48. Messenger, P. M., \& Smith, G. S. (2010). Cultural heritage management: A global perspective. Gainesville, FL: University Press of Florida.CrossRef (http://dx.doi.org/10.5744/florida/9780813034607.001.0001)Google Scholar (http://scholar.google.com/scholar_lookup?

title=Cultural\%2oheritage\%20management\%3A\%20A\%2oglobal\%2operspective\&a uthor=PM.\%20Messenger\&author=GS.\%20Smith\&publication_year=2010)

49. Müller, D. K., \& Jansson, B. (2007a). Tourism in peripheries: Perspectives from the far north and south. Wallingford, UK: Cabi.CrossRef (http://dx.doi.org/10.1079/9781845931773.0000)Google Scholar (http://scholar.google.com/scholar_lookup?

title=Tourism\%20in\%2operipheries\%3A\%20Perspectives\%2ofrom\%2othe\%2ofar \%20north\%20and\%20south\&author=DK.\%20M\%C3\%BCller\&author=B.\%2OJanss on\&publication_year $=2007$ )

50. Müller, D. K., \& Jansson, B. (2007b). The difficult business of making pleasure peripheries prosperous: Perspectives on space place and environment. In D. K. Müller \& B. Jansson (Eds.), Tourism in peripheries: Perspectives from the far North and South (pp. 3-18). Wallingford, UK: Cabi.CrossRef (http://dx.doi.org/10.1079/9781845931773.0003)Google Scholar (http://scholar.google.com/scholar_lookup? title=The\%2odifficult\%2obusiness\%20of\%20making\%2opleasure\%2operipheries \%2oprosperous\%3A\%20Perspectives\%20on\%20space\%2oplace\%20and\%20enviro nment\&author=DK.\%20M\%C3\%BCller\&author=B.\%20Jansson\&pages=318\&publication_year=2007)

51. Neuhofer, B., Buhalis, D., \& Ladkin, A. (2015). Smart technologies for personalized experiences: A case study in the hospitality domain. Electronic Markets, 25(1), 243-254. doi:10.1007/s12525-015-0182-1 (http://dx.doi.org/10.1007/s12525-0150182-1).CrossRef (http://dx.doi.org/10.1007/s12525-015-0182-1)Google Scholar (http://scholar.google.com/scholar_lookup?

title=Smart\%20technologies\%2ofor\%2opersonalized\%2oexperiences\%3A\%20A\%2 ocase\%20study\%20in\%20the\%2ohospitality\%2odomain\&author=B.\%20Neuhofer \&author=D.\%20Buhalis\&author=A.\%2OLadkin\&journal=Electronic\%20Markets\&v olume $=25 \&$ issue $=1 \&$ pages $=243$ -

254\&publication_year $=2015 \&$ doi $=10.1007 \% 2 F s 12525-015-0182-1)$

52. Nuryanti, W. (1996). Heritage and postmodern tourism. Annals of Tourism

Research, 23(2), 249-260. doi:10.1016/0160-7383(95)ooo62-3

(http://dx.doi.org/10.1016/0160-7383(95)oo062-3).CrossRef

(http://dx.doi.org/10.1016/0160-7383(95)ooo62-3)Google Scholar

(http://scholar.google.com/scholar_lookup?

title=Heritage\%20and\%2opostmodern\%20tourism\&author=W.\%2oNuryanti\&jour nal=Annals $\% 200 f \% 20$ Tourism $\% 20$ Research $\&$ volume $=23 \&$ issue $=2 \&$ pages $=249$ 260\&publication_year $=1996 \&$ doi $=10.1016 \% 2 \mathrm{Fo160}-7383 \% 2895 \% 2900062-3$ )

53. Olick, J. K., Vinitzky-Seroussi, V., \& Levy, D. (Eds.). (2011). The collective memory reader. New York, NY: Oxford University Press.Google Scholar (http://scholar.google.com/scholar_lookup? title=The\%20collective\%20memory\%20reader\&publication year=2011)

54. Page, S., \& Hall, C. M. (2003). Managing urban tourism. Harlow, UK: Prentice Hall.Google Scholar (http://scholar.google.com/scholar_lookup? title=Managing\%20urban\%20tourism\&author=S.\%20Page\&author=CM.\%20Hall\& publication year $=2003$ )

55. Pahl, N., \& Richter, A. (2007). SWOT analysis: Idea, methodology and a practical approach. Munchen, DE: GRIN Verlag.Google Scholar

(http://scholar.google.com/scholar_lookup?

title=SWOT\%20analysis\%3A\%20Idea\%2C\%2omethodology\%2oand\%20a\%2oprac 
tical\%20approach\&author=N.\%20Pahl\&author=A.\%2oRichter\&publication_year= 2007)

56. Pearce, D. G. (2002). Tourism today: A geographical analysis (2nd ed.). Harlow Essex, UK: Longman Scientific \& Technical.Google Scholar

(http://scholar.google.com/scholar_lookup?

title=Tourism\%20today\%3A\%20A\%20geographical\%20analysis\&author=DG.\%20 Pearce\&publication_year=2002)

57. Plog, S. (1974). Why destination areas rise and fall in popularity. Cornell Hotel and Restaurant Administration Quarterly, 42(3), 13-24.CrossRef (http://dx.doi.org/10.1016/So010-8804(01)81020-X)Google Scholar (http://scholar.google.com/scholar_lookup? title=Why\%2odestination\%20areas\%2orise\%20and\%2ofall\%2oin\%2opopularity\& author $=$ S.\%20Plog\&journal =Cornell\%20Hotel\%20and\%20Restaurant\%2OAdminis tration\%20Quarterly\&volume $=42 \&$ issue $=3 \&$ pages $=13-24 \&$ publication $\_$year $=1974$ )

58. Plog, S. (2001). Why destination areas rise and fall in popularity: An update of a Cornell Quarterly class. Cornell Hotel and Restaurant Administration Quarterly, 14(4), 55-58.CrossRef (http://dx.doi.org/10.1177/001088047401400409)Google Scholar (http://scholar.google.com/scholar lookup?

title=Why\%2odestination\%20areas\%2orise\%20and\%2ofall\%20in\%2opopularity\% 3A\%20An\%20update\%20of\%20a\%20Cornell\%20Quarterly\%2oclass\&author=S.\%2 oPlog\&journal=Cornell\%20Hotel\%20and\%2oRestaurant\%20Administration\%20Q uarterly\&volume $=14$ \&issue $=4$ \&pages $=55-58$ \&publication year $=2001$ )

59. Prats, L. (1997). Antropología y patrimonio. Barcelona, ES: Ariel.Google Scholar (http://scholar.google.com/scholar_lookup?

title=Antropolog\%C3\%ADa\%20y\%20patrimonio\&author=L.\%20Prats\&publication year $=1997)$

60. Rusalić, D. (2009). Making the intangible tangible: The new interface of cultural heritage. Belgrade, RS: Institute of Ethnography SASA.Google Scholar (http://scholar.google.com/scholar_lookup?

title=Making\%20the\%2ointangible\%2otangible\%3A\%20The\%2onew\%2ointerface \%20of\%20cultural\%2oheritage\&author=D.\%20Rusali\%C4\%87\&publication_year= 2009)

61. Ryan, M.-L. (2004). Multivariant narratives. In S. Schreibman, R. G. Siemens \& J. Unsworth (Eds.), A companion to digital humanities (pp. 415-430). Malden, MA: Blackwell Pub.CrossRef (http://dx.doi.org/10.1002/9780470999875.ch28)Google Scholar (http://scholar.google.com/scholar_lookup? title=Multivariant\%20narratives\&author $=$ M-L.\%20Ryan\&pages $=415^{-}$ 430\&publication_year=2004)

62. Salazar, J. F., \& Bushell, R. (2013). Heritage for sale: The misrepresentation of voice in northern Chile. In R. Staiff, R. Bushell \& S. Watson (Eds.), Heritage and tourism: Place, encounter, engagement (pp. 187-212). London, UK: Routledge; Taylor \& Francis Group.Google Scholar (http://scholar.google.com/scholar_lookup?

title=Heritage\%20for\%20sale\%3A\%20The\%20misrepresentation\%20of\%20voice\% 20in\%20northern\%20Chile\&author=JF.\%20Salazar\&author=R.\%20Bushell\&pages $=187-212 \&$ publication_year $=2013$ )

63. Schensul, S. L., Schensul, J. J., \& LeCompte, M. D. (1999). Essential ethnographic methods: Observations, interviews, and questionnaires (1st ed.). Walnut Creek, CA: AltaMira Press.Google Scholar (http://scholar.google.com/scholar lookup? title=Essential\%20ethnographic\%2omethods\%3A\%20Observations\%2C\%2ointervi ews\%2C\%20and\%20questionnaires\&author=SL.\%2OSchensul\&author=JJ.\%20Sch ensul\&author=MD.\%20LeCompte\&publication_year=1999)

64. Smith, M. K., \& Richards, G. (Eds.). (2013). The Routledge handbook of cultural tourism. New York, NY: Routledge.Google Scholar

(http://scholar.google.com/scholar_lookup?

title=The\%20Routledge\%2ohandbook\%20of\%20cultural\%2otourism\&publication year $=2013$ )

65. Staiff, R., Bushell, R., \& Watson, S. (Eds.). (2013). Heritage and tourism: Place, encounter, engagement. London, UK: Routledge.Google Scholar

(http://scholar.google.com/scholar_lookup?

title=Heritage \%20and\%20tourism\%3A\%20Place\%2C\%2oencounter\%2C\%2oengag ement\&publication_year=2013)

66. Stanco, F., Battiato, S., \& Gallo, G. (Eds.). (2011). Digital imaging for cultural heritage preservation: Analysis, restoration, and reconstruction of ancient artworks. Boca Raton, FL: CRC Press.Google Scholar (http://scholar.google.com/scholar_lookup?

title=Digital\%2oimaging\%20for\%20cultural\%2oheritage\%2opreservation\%3A\%20 Analysis\%2C\%2orestoration\%2C\%20and\%2oreconstruction\%20of\%20ancient\%20 artworks\&publication_year=2011)

67. Timothy, D. J., \& Boyd, S. W. (2003). Heritage tourism (1st ed.). New York, NY: Prentice Hall.Google Scholar (http://scholar.google.com/scholar_lookup? title $=$ Heritage\%20tourism\&author=DJ.\%20Timothy\&author $=$ SW.\%20Boyd\&publi cation_year=2003)

68. Turner, L., \& Ash, J. (1976). The Golden hordes: International tourism and the pleasure periphery (2nd ed.). New York, NY: St. Martin's Press.Google Scholar (http://scholar.google.com/scholar_lookup?

title=The\%2oGolden\%2ohordes\%3A\%20International\%2otourism\%20and\%2othe \%20pleasure\%2operiphery\&author=L.\%20Turner\&author=J.\%20Ash\&publication _year=1976) 
69. Tussyadiah, I., \& Inversini, A. (Eds.). (2015). Information and communication technologies in tourism 2015: Proceedings of the international conference in Lugano, Switzerland, February 3-6, 2015. Cham, DE: Springer.

70. Wachowiak, H. (2006). Tourism and borders: Contemporary issues, policies, and international research. Aldershot, England: Ashgate.Google Scholar (http://scholar.google.com/scholar_lookup? title=Tourism\%20and\%2oborders\%3A\%20Contemporary\%2oissues\%2C\%2opolici es\%2C\%20and\%2ointernational\%2Oresearch\&author=H.\%2OWachowiak\&publicat ion_year=2006)

71. Wall, G. (2000). Centre-Periphery. In J. Jafari (Ed.), Encyclopedia of tourism (p. 76). London, UK: Routledge.Google Scholar (http://scholar.google.com/scholar lookup?title=CentrePeriphery\&author $=$ G.\%20Wall\&pages $=76 \&$ publication_year $=2000$ )

72. Wanhill, S. (1997). Peripheral area tourism: A European perspective. Progress in Tourism and Hospitality Research, 3(1), 47-70. doi:10.1002/(SICI)10991603(199703)3:1<47:AID-PTH38>3.0.CO;2-F

(http://dx.doi.org/10.1002/(SICI)1099-1603(199703)3\%3A1\%3c47\%3AAIDPTH38\%3e3.o.CO\%3B2-F).CrossRef (http://dx.doi.org/10.1002/(SICI)10991603(199703)3\%3A1<47\%3A\%3AAID-PTH38>3.0.CO\%3B2-F) Google Scholar (http://scholar.google.com/scholar lookup?

title=Peripheral\%20area\%20tourism\%3A\%20A\%20European\%20perspective\&aut hor=S.\%20Wanhill\&journal=Progress\%20in\%2oTourism\%20and\%2oHospitality\% 20Research\&volume $=3$ \&issue $=1 \&$ pages $=47$ -

70\&publication_year $=1997 \&$ doi $=10.1002 \% 2$ F\%28SICI\%291099-

1603\%28199703\%293\%3A1\%3C47\%3A\%3AAID-PTH38\%3E3.0.CO\%3B2-F)

73. Wang, D., \& Xiang, Z. (2012). The new landscape of travel: A comprehensive analysis of smartphone apps. In M. Fuchs, F. Ricci, \& L. Cantoni (Eds.), International conference on information and communication technologies in tourism, Information and communication technologies in tourism 2012: Proceedings of the international conference in Helsingborg, Sweden, January 2527, 2012 (pp. 308-319). Vienna, New York: Springer.

74. Waterton, E., \& Watson, S. (Eds.). (2015). The Palgrave handbook of contemporary heritage research. Hampshire, UK: Palgrave Macmillan.Google Scholar (http://scholar.google.com/scholar_lookup? title=The\%20Palgrave\%2ohandbook\%20of\%20contemporary\%2oheritage\%2orese arch\&publication_year=2015)

75. Weaver, D. (2005). The distinctive dynamics of exurban tourism. International Journal of Tourism Research, 7(1), 23-33. doi:10.1002/jtr.521 (http://dx.doi.org/10.1002/jtr.521).CrossRef (http://dx.doi.org/10.1002/jtr.521)Google Scholar (http://scholar.google.com/scholar lookup? title=The\%20distinctive\%20dynamics\%20of\%20exurban\%2otourism\&author=D.\% 20Weaver\&journal=International\%20Journal\%20of\%20Tourism\%20Research\&vo lume $=7 \&$ issue $=1 \&$ pages $=23-33 \&$ publication_year $=2005 \&$ doi $=10.1002 \% 2 F j t r .521$ )

76. West, S. (2010). Understanding heritage in practice. Manchester: Manchester University Press, Open University.Google Scholar (http://scholar.google.com/scholar_lookup?

title=Understanding\%2oheritage\%20in\%2opractice\&author=S.\%20West\&publicati on_year=2010)

77. White, R., Engelen, G., \& Uljee, I. (2015). Modeling cities and regions as complex systems: From theory to planning applications. Cambridge, MA: MIT

Press.CrossRef

(http://dx.doi.org/10.7551/mitpress/9780262029568.001.0001)Google Scholar (http://scholar.google.com/scholar_lookup?

title=Modeling\%2Ocities\%20and\%2oregions\%20as\%2ocomplex\%20systems\%3A\% 20From\%20theory\%20to\%2oplanning\%20applications\&author=R.\%20White\&aut hor $=$ G.\%20Engelen\&author $=$ I.\%20Uljee\&publication_year $=2015$ )

78. Williams, J., \& Lawson, R. (2001). Community issues and resident opinions of tourism. Annals of Tourism Research, 28(2), 269-290. doi:10.1016/So1607383(oo)ooo3o-X (http://dx.doi.org/10.1016/So160-7383(00)ooo30-

X).CrossRef (http://dx.doi.org/10.1016/S0160-7383(00)00030-X)Google Scholar (http://scholar.google.com/scholar_lookup?

title=Community\%20issues\%20and\%20resident\%20opinions\%20of\%20tourism\&a uthor=J.\%20Williams\&author=R.\%20Lawson\&journal=Annals\%20of\%20Tourism $\% 20$ Research $\&$ volume $=28 \&$ issue $=2 \&$ pages $=269$ 290\&publication year $=2001 \&$ doi $=10.1016 \% 2 F S 0160-7383 \% 2800 \% 2900030-X$ )

79. Williams, S. (1998). Tourism geography (1st ed.). London, UK: Routledge.Google Scholar (http://scholar.google.com/scholar_lookup? title=Tourism\%20geography\&author=S.\%20Williams\&publication_year=1998)

80. Xiang, Z., Tussyadiah, I., \& Buhalis, D. (2015a). Smart destinations: Foundations, analytics, and applications. Journal of Destination Marketing \& Management, 4(3), 143-144. doi:10.1016/j.jdmm.2015.07.001

(http://dx.doi.org/10.1016/j.jdmm.2015.07.001).CrossRef

(http://dx.doi.org/10.1016/j.jdmm.2015.07.001)Google Scholar

(http://scholar.google.com/scholar_lookup?

title $=$ Smart $\%$ 20destinations $\% 3 \mathrm{~A} \% 2$ Foundations $\% 2 \mathrm{C} \%$ 20analytics $\% 2 \mathrm{C} \% 2 \mathrm{Oand} \%$ 20applications\&author=Z.\%20Xiang\&author=I.\%20Tussyadiah\&author=D.\%20Bu halis\&journal=Journal\%20of\%20Destination\%20Marketing\%20\%26\%20Managem ent\&volume $=4$ \&issue $=3$ \&pages $=143-$

$144 \&$ publication_year $=2015 \&$ doi $=10.1016 \% 2 F j . j d m m .2015 .07 .001)$ 
81. Xiang, Z., Wang, D., O’Leary, J. T., \& Fesenmaier, D. R. (2015b). Adapting to the Internet: Trends in travelers' use of the web for trip planning. Journal of Travel Research, 54(4), 511-527. doi:10.1177/0047287514522883

(http://dx.doi.org/10.1177/0047287514522883).CrossRef

(http://dx.doi.org/10.1177/0047287514522883)Google Scholar

(http://scholar.google.com/scholar lookup?

title=Adapting\%20to\%20the\%2OInternet\%3A\%2oTrends\%2oin\%2otravelers\%E2 \%80\%99\%20use\%20of\%2othe\%20web\%20for\%20trip\%2oplanning\&author=Z.\%2 oXiang\&author=D.\%20Wang\&author $=J$ T.\%20O\%E2\%80\%99Leary\&author $=$ DR.\% 20Fesenmaier\&journal=Journal\%20of\%20Travel\%20Research\&volume=54\&issue $=4 \&$ pages $=511-527 \&$ publication $\_$year $=2015 \&$ doi $=10.1177 \% 2$ Foo 47287514522883$)$

\section{Copyright information}

(C) Springer International Publishing AG 2017

\section{About this paper}

- Publisher Name Springer, Cham

- Print ISBN 978-3-319-47731-2

- Online ISBN 978-3-319-47732-9

- eBook Packages Business and Management

- About this book

- $\underline{\text { Reprints and Permissions }}$

\section{SPRINGER NATURE}

(C) 2017 Springer International Publishing AG. Part of Springer Nature.

Not logged in Not affiliated 95.95.71.58 